
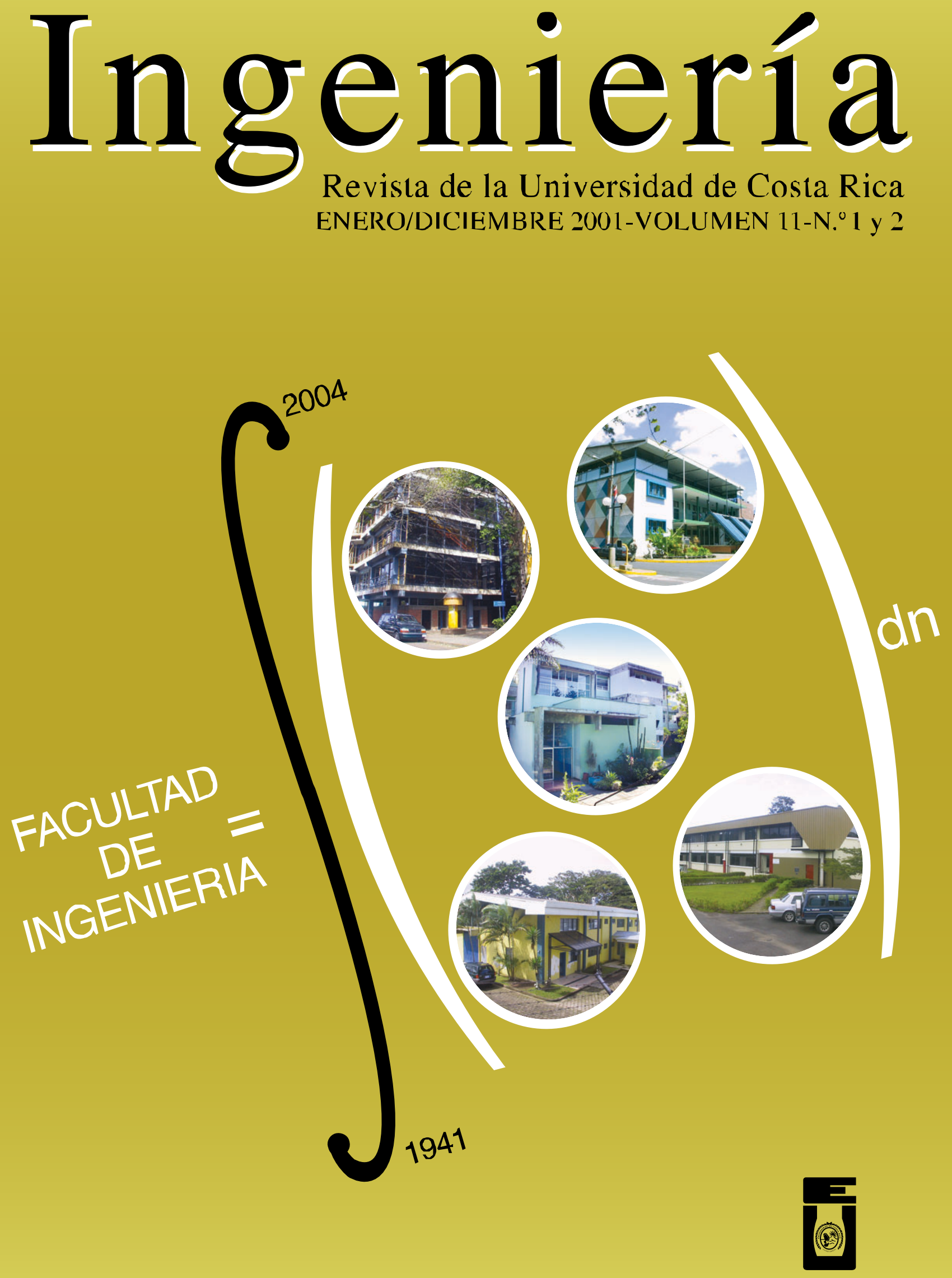


\section{Trabajos de Graduación 2000}





\section{TRABAJOS FINALES DE GRADUACIÓN DE LA ESCUELA DE INGENIERÍA ELÉCTRICA 2000}

\section{Referencias bibliográficas}

\section{Licenciatura}

Hidalgo Moreira, Francisco; Soto Montero, Mauricio.

Estudio y análisis de un modelo Coteh para aumentar el índice de confiabilidad y los ingresos económicos en una empresa de distribución eléctrica. ( Se efectuó en la empresa de Servicios Publicos de Heredia S. A.)

Ingeniería Eléctrica -San José CR.

F. Hidalgo M; M. Soto M. 2000

Acosta Guevara, Hahaziel; Alvarado Contreras, Tito José

Diseño de una red de sistemas contraincendios interconectados mediante fibra óptica y controlados remotamente por un PC.

Ingeniería Eléctrica -San José, CR.

J. Acosta G; T. Alvarado C. 2001

Chavarria Corella, Manuel

Mallas de puesta a tierra.

Ingeniería Eléctrica -San José, CR.

M. Cavarria C. 2000

Rodríguez Castro, Oscar Guillermo

Monitorizar y diagnosticar sistemas de energía para centrales de telecomunicaciones.

Ingeniería Eléctrica -San José, CR.

O. Rodríguez C. 2000

Gomes Umaña, Egides; Sibaja Amador, Mauricio.

Confección de los planos eléctricos para las siete geometrías y diseño de la iluminación del área de "picnic" del Parque Nacional de Diversiones.
Ingeniería Eléctrica -San José, CR.

E. Gómez U; M. Sibaja A. 2000

Rivera Lamsick, Helmut.

Análisis del sistema de control de una turbina, basado en el gobernador Mprea DGC Guuc.

Ingeniería Eléctrica -San José, CR.

H. Rivera L. 2000

Starcevic Rivera, Aldo.

Diseño eléctrico urbano.

Ingeniería Eléctrica -San José, CR.

A. Starcevic R. 2000

Bertin Prado, Roberto; Kopper Barquero, Luis Diego.

Análisis y diseño de la red ATM de Racsa.

Ingeniería Eléctrica -San José, CR.

R. Bertin P; L. Kopper B. 2000

\section{Bachillerato}

Mc Parlane Gomez, Juan Carlos

Desarrollo de una estrategia PID para el control automático de nivel utilizando el PLC SLC5/05 Ingeniería Eléctrica -San José CR.

J Mc Parlane G, 2000

Araya Vargas, José Julián

Desarrollo de una interfaz humana máquina para acabar dispositivos de control de energía de Cutler-Hammer

Ingeniería Eléctrica -San José, CR.

J. Araya V. 2000

Salazar Castro, Gustavo

Estudio sobre el aumento de la capacidad de transporte de energía eléctrica mediante el cambio de conductor en la L.T. Siquirres-Moín 
Ingeniería Eléctrica -San José, CR.

G. Salazar C. 2000

Saenz Fallas, Roberto

Desarrollo de un lazo de control digital directo a través del puerto serie.

Ingeniería Eléctrica -San José, CR.

R. Saenz F. 2000

Araya León, José Manuel.

Desarrollo de una HMI de la planta de tratamiento de aguas residuales de la empresa CutlerHammer.

Ingeniería Eléctrica -San José, CR.

J. Araya L. 2000

Marín González, Marisela; Campos Artavia Christian

Manual de utilización de la máquina QUICKCIRCUIT

Ingeniería Eléctrica -San José, CR.

M Marín G; C. Campos A. 2000

Murillo Fallas, Agustín

La problemática de la aplicación de las redes de distancia en la protección de redes de transmisión que presentan configuraciones complejas.

Ingeniería Eléctrica -San José, CR.

A. Murillo F. 2000

Valerio Mena, Christian

Evaluación de la instalación de pararrayos en la línea de transmisión Arenal-Barranca para mejorar el desempeño ante descargas atmosféricas.

Ingeniería Eléctrica -San José, CR.

C. Valerio M. 2000

Venegas Garro, Richard.

Funcionamiento de una antena satélite SCPC (single channel per corrier)

Ingeniería Eléctrica -San José, CR.

R. Venegas G. 2000

Ramírez Alvarado, Kevin

Cálculo del precio en barra para el mercado eléctrico centroamericano.

Ingeniería Eléctrica -San José, CR.

K. Alvarado R. 2000
Rivera Alvarado, Javier; Avendaño Torres, Felipe Problemática del ruido electromagnético en la industria.

Ingeniería Eléctrica -San José, CR.

J. Rivera A.; F. Torres A. 2000

González Fallas, Ernesto.

Probador de tarjetas AI-TDR (PROAI-TDB)

Ingeniería Eléctrica -San José, CR.

E. Gonzalez F. 2000

Halabi García, Mayid; Salazar Viales, Randall.

Normas y metodologías para el diseño de redes de cableado estructurado.

Ingeniería Eléctrica -San José, CR.

M. Halabi G; R. Salazar V. 2000

Sancho Chaves, Rolando.

Desarrollo de un programa para el cálculo de parámetros de líneas de transmisión dependientes de la frecuencia.

Ingeniería Eléctrica -San José, CR.

R. Sancho C. 2000

Castro Zamora, Mario.

Análisis de la eficiencia del grupo turbogenerador en la planta geotérmica Miravalles II.

Ingeniería Eléctrica -San José, CR.

M. Castro Z. 2000

Rojas Arce, Alejandro José.

Estimación de la silueta de objetos reales en movimiento mediante un detector de cambios de máxima verosimilitud a partir de la información proveniente de una cámara monocular.

Ingeniería Eléctrica -San José, CR.

A. Rojas A. 2000

Aguilar Corrales, Vilma.

Análisis de las diferencias entre la CPU de un PLC y un computador Industrial con Win Ce en control. Ingeniería Eléctrica -San José, CR.

V. Aguilar C. 2000

Blanco Benevides, Sergio.

Simulación de algoritmos para la cancelación del eco.

Ingeniería Eléctrica -San José, CR.

S. Blanco B. 2000 
Muñoz Castro, Mauricio.

Sistemas de comunicación celular en Costa Rica. Ingeniería Eléctrica -San José, CR.

M. Muñoz C. 2000

García González, Luis.

Implementación de un enrutador utilizando el sistema operativo Linux.

Ingeniería Eléctrica -San José, CR.

L. García G. 2000

Murillo Agüero, Rolando.

Diseño y montaje de un controlador discreto de posición para el carrusel de la celda de manufactura, utilizando el microcontrolador 8051 .

Ingeniería Eléctrica -San José, CR.

R. Murillo A. 2000

Chavarría Gamboa, Nelson; Troyo Rodríguez, Francisco.

Montaje y pruebas de un sistema de alarma de intrusión utilizando láser.

Ingeniería Eléctrica -San José, CR.

N. Chavarría G; F. Troyo R. 2000

Novo Gutiérrez, Francisco.

Diseño del control de flujo de amoniaco para enfriamiento de doce tanques de almacenamiento de cerveza por medio de un PLC en la Cervecería Costa Rica. S.A.

Ingeniería Eléctrica -San José, CR.

F. Novo G. 2000

Ávila Duarte, Mauricio.

Control de motor de corriente directa usando MATLAB/SIMULINK.

Ingeniería Eléctrica -San José, CR.

M. Avila D. 2000.

Alvarado Quesada, Federico.

Identificación paramétrica para una planta hidráulica de nivel.

Ingeniería Eléctrica -San José, CR.

F. Alvarado Q. 2000.

Pacheco Vázquez, Melvin.

Construcción de un interfaz gráfica para el diseño de controladores robustos.

Ingeniería Eléctrica -San José, CR. 2000
Apu Apu, Alejandro.

Análisis del recurso eólico de San Jerónimo de Moravia.

Ingeniería Eléctrica -San José, CR.

A. Apu A. 2000

Badilla Salas, Gustavo; Soto Leandro, Felipe. Auditoría energética de Industrias Cerdas S.A. Ingeniería Eléctrica -San José, CR.

G. Badilla S.; F. Soto L. 2000

Garro Calvo, Michael.

Puesta en marcha del toolbox de tiempo real de MATLAB para la implementación de un control PID para un proceso industrial.

Ingeniería Eléctrica -San José, CR.

M. Garro C. 2000.

Murillo Fuentes, Carlos Jaime.

Diseño de ecualizadores digitales por medio de una interfaz gráfica del usuario.

Ingeniería Eléctrica -San José, CR.

C. Murillo F. 2000

Benavides Murillo, Francisco José.

Codificación de una señal discreta en subbandas: aplicación de las onduletas.

Ingeniería Eléctrica -San José, CR.

F. Benavides M. 2000

Ramírez Abrahams, Francisco Jesús

Bases de la programación y optimización de un proceso en una celda de manufactura, por medio de un PLC y un computador personal.

Ingeniería Eléctrica -San José, CR.

F. Ramírez A. 2000

Zeledón Mendez, Peter Bernal, Murillo Calvo, Manrique

Reparación del brazo hidráulico HRA934 y desarrollo de hardware y software para su control y utilización en conjunto con una banda transportadora automatizada para implementar un proceso

Ingeniería Eléctrica -San José, CR.

P. Zeledon M, M. Murillo C. 2000

Elizondo Orozco, Homer

Apertura y regulación de las telecomunicaciones 
en Costa Rica

Ingeniería Eléctrica -San José, CR.

H. Elizondo O. 2000

Venegas Zuñiga, Gustavo

Diseño de una interfaz humano-máquina para algunos miembros de la familia de instrumentos de administración de energía de Cuttler-Hammer Ingeniería Eléctrica -San José, CR.

G. Venegas Z. 2000

Bogantes Rojas, Graciela; Alfaro Montero, David

Coordinación y ajuste de la protección de impedancia de la línea Río Macho-San Miguel 230 KV Ingeniería Eléctrica -San José, CR.

G. Bogantes R; D. Alfaro M. 2000

Arroyo Chaves, Ismael

Implementación de un sistema hidráulico, su identificación paramétrica y el desarrollo de un sistema de control robusto

Ingeniería Eléctrica -San José, CR.

I. Arroyo C. 2000

\section{Resúmenes de las tesis sobresalientes}

Zeledón Méndez, Peter Bernal, Murillo Calvo, Manrique

'Reparación del brazo hidráulico HRA934 y desarrollo de hardware y software para su control y su utilización en conjunto con una banda transportadora automatizada para implementar un proceso"

Ingeniería Eléctrica -San José, CR.

P. Zeledón M, M. Murillo C. 2000

Se desea poner a funcionar el brazo HRA934 con el fin de que los estudiantes puedan desarrollar procesos a pequeña escala, permitiéndoles una mejor visión y acercamiento al área de la automatización industrial.

Se deberá proceder a reparar el sistema hidráulico, mecánico y eléctrico del robot.
En lo referente al sistema eléctrico se utilizarán todas aquellas etapas originales que siguen siendo funcionales y que por su naturaleza no se pueden cambiar o mejorar, pero las que debido al tiempo y a los cambios tecnológicos han quedado rezagadas, se remplazarán.

Se diseñará un interfaz que permita la comunicación entre un micro-controlador y el HRA934. Se desarrollará también un programa a través del cual el estudiante pueda manejar de forma manual o automática el robot que le permita crear sus propias rutinas a través de un computador personal.

Se construirá una banda transportadora automatizada con el fin de que trabaje en forma conjunta con el brazo hidráulico.

Acuña Córdoba, Andrés Alonso, Zúñiga Pérez, Erick Francisco.

"Compresión de imágenes estáticas mediante la utilización de la transformada discreta de Wavelet"

Ingeniería Eléctrica-San José, CR.

A.. Acuña C, E. Zúñiga P. 2000

Este trabajo de compresión de imágenes es un primer acercamiento a la compresión de imágenes por medio de la utilización de una transformada en la que se aplican todas las partes con que consta un compresor.

El objetivo principal es demostrar que si se utiliza la DWT en la compresión de una imagen se obtiene un desempeño mejor que cuando se utiliza la DCT, la cual es utilizada en el estándar de compresión de imágenes JPEG.

Para llevar a cabo este trabajo, primero se hizo un exhaustivo estudio sobre wavelets y compresión de imágenes en la literatura especializada. Seguidamente, se realizó un estudio de la característica de los diferentes filtros wavelets más populares para compresión de imágenes. Luego, entre esos filtros y de acuerdo con sus caracte- 
rísticas, se escogieron unos filtros para realizar pruebas de compresión. Después se tomaron los algoritmos DWT en dos dimensiones presentes en la literatura y se implemento en el lenguaje de programación Matlab, en tanto que la realización de la DCT se efectuó con una función propia del Matlab. Una vez hecha la implementación, se busco en la literatura un algoritmo de codificación, que fuera posible utilizar tanto con la DCT como con la DWT.

El resultado más importante fue que los grados de compresión obtenidos con DWT fueron mejores que los obtenidos con la DCT, tanto en la razón de compresión como en la calidad de la imagen. La calidad de la imagen reconstruida se evaluó empleando la potencia de la relación señal a ruido (PSNR)

Dentro de las conclusiones principales encontramos que la razón de compresión y calidad de la imagen reconstruida varía de acuerdo a la imagen procesada y a la transformación utilizada para una misma razón de compresión.

La compresión mediante DCT introduce una cantidad significativa de ruido, mientras que con la DWT no ocurre lo mismo. 
